\title{
Improve accuracy for automatic acetabulum segmentation in CT images
}

\author{
Hao Liu ${ }^{\mathrm{a}, \mathrm{b}}$, Jianning Zhao ${ }^{\mathrm{b}}$, Ning Dai ${ }^{\mathrm{a}}$, Hongbo Qian ${ }^{\mathrm{b}}$ and Yuehong Tang ${ }^{\mathrm{a},{ }^{*}}$ \\ ${ }^{a}$ Nanjing University of Aeronautics and Astronautics, P.R. China \\ ${ }^{b}$ Jinling Hospital, Department Orthopedics, Nanjing University School of Medicine, P.R. China
}

\begin{abstract}
Separation of the femur head and acetabulum is one of main difficulties in the diseased hip joint due to deformed shapes and extreme narrowness of the joint space. To improve the segmentation accuracy is the key point of existing automatic or semi-automatic segmentation methods. In this paper, we propose a new method to improve the accuracy of the segmented acetabulum using surface fitting techniques, which essentially consists of three parts: (1) design a surface iterative process to obtain an optimization surface; (2) change the ellipsoid fitting to two-phase quadric surface fitting; (3) bring in a normal matching method and an optimization region method to capture edge points for the fitting quadric surface. Furthermore, this paper cited vivo CT data sets of 40 actual patients (with 79 hip joints). Test results for these clinical cases show that: (1) the average error of the quadric surface fitting method is $2.3(\mathrm{~mm}) ;(2)$ the accuracy ratio of automatically recognized contours is larger than $89.4 \%$; (3) the error ratio of section contours is less than $10 \%$ for acetabulums without severe malformation and less than $30 \%$ for acetabulums with severe malformation. Compared with similar methods, the accuracy of our method, which is applied in a software system, is significantly enhanced.
\end{abstract}

Keywords: Acetabulum, segmentation, ellipsoid fitting, edge points, computer tomography

\section{Introduction}

Total hip replacement (THR) is a common orthopedic procedure in which many digitization methods are extensively used at present to improve its operation quality, e.g. finite element analysis, $\mathrm{CAD} / \mathrm{CAM}$ for prosthesis, virtual surgery, computer aided surgery, etc [1]. Because 3D digitization models of hip joints, which are the enarthrosis consisting of the femur head and the acetabulum, are foundations of these digitization methods [2,3], close attention is paid to automatic segmentation and automatic 3D reconstruction in THR digitization techniques. As the joint space between the femur head and the acetabulum is very small, it usually becomes obscure and even disappears in CT images due to factors such as lesion, malformation, damage, image resolution and scan posture. Consequently, it is a challenging task to segment the femur head and the acetabulum from CT images with high automatic degree and accuracy.

Although there have been many algorithms to segment medical images [4], no one can automatically segment the hip joint all alone with right acetabulum surface in every slice. The existing algorithms could be divided into the following categories: (1) pixel clustering method [5-8]. Threshold segmenta-

\footnotetext{
${ }^{*}$ Corresponding author: Yuehong Tang, Nanjing University of Aeronautics and Astronautics, P.R. China. Tel.: 02584895781; Fax: 025-84895781; E-mail: tangyuehong@nuaa.edu.cn.
}

0959-2989/14/\$27.50 @ 2014 - IOS Press and the authors. 
tion and region growth are usually used in the method whose main disadvantage is leakage [9]. Different from threshold and region growth methods, Cheng et al.'s method [8] employ valley emphasis and the Bayes decision rules. Though several algorithms are combined and the segmentation effect is improved, Cheng et al.'s method is still difficult to obtain right region for hips and femurs in any slice. They avoid the disadvantage by using three methods: (i) fill holes; (ii) remove noises; (iii) pixel heuristics. Because sizes of the filling diamond and noise region have to be manually given, and because pixels of acetabulum surfaces are probably un-connected in adjacent slices, their method probably fails to obtain correct contours for hips and femurs in some slices. (2) heuristic method [10-14]. This method extracts contours by using active contour models (ACM) slice by slice on condition that contours in the previous slice have been accurately extracted. There are several kinds of ACM: the edge-based ACM, the region-based ACM and the integrated ACM [15]. The method suffers from two disadvantages: (i) contour leakage problem [10] and (ii) errors diffusing, i.e., the accuracy of previous contours affects the accuracy of current contours. Such two disadvantages lead to low automation degree of the method; (3) prior information method [16-21]. This method estimates the shape information of hip joints in advance, which is automatically inputted to the segmentation program as the known conditions. There have been several kinds of prior knowledge: (i) the shape of femur head (or acetabulum). For example, Zoroofi et al. [16,17] fit the femur head as an ellipsoid. However, their fitting ellipsoid is not accurate because of the high error ratio [16]. In [18], the femur head is fitted by a sphere, which is hard to be corrected on account of the large fitting error in some slices; (ii) Atlas of hip joints $[19,20]$. These atlases consist of labeled CT data sets and associated anatomical point landmarks. The task of segmentation is to transfer the atlas information to patient data. (iii) statistical model method [21]. This method has to construct a statistical model in advance and then register and deform the statistical model in new CT data.

Due to the complexity of the shape of tissues and imbalance of gray distribution in CT images, it is impossible to obtain accurate segmentation results by automatic methods without prior information [22]. Although atlases and statistical models can be regarded as prior information, by which it is difficult to achieve a segmentation method with supermatic and high-accuracy owing to shape-oriented differences of individuals and finiteness of specimen. As a result, our method uses an iterative process to obtain an optimum ellipsiod. When the ellipsiod captures edge points of the acetabulum, a normalbased optimization region selection method is used. The method can intelligentializedly avoid noise disturbances. High fitting accuracy and low noise disturbances are two main advantages of our algoritm. Main contributions of our algoritm include: (1) design an iterative process to obtain the optimum fitting surface; (2) design a two-step method to fit ellipsiods; (3) design a normal-based optimization region selection method to capture edge points.

\section{Materials and methods}

\subsection{Classification of data sets}

We have collected 40 sets (80 hip joints) in-vivo CT data of actual patients, while one of them has completed the THR operation. Hence, there are data-sets of 79 hip joints remaining to be studied. These data-sets were accumulated between 2011 and 2013 at Nanjing Jinling Hospital from patients suffering from complications such as pain, inflammation, and other symptoms in their hip joints. The CT data-sets have been scanned by the Siemens dual-source CT machine. The number of slices of per data 


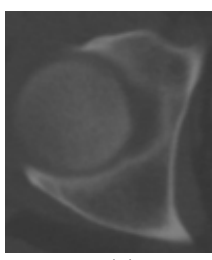

(a)

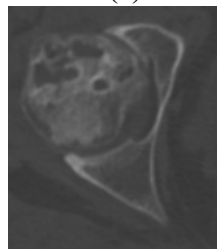

(g)

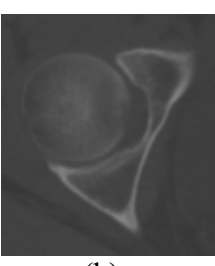

(b)

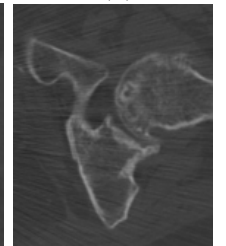

(h)

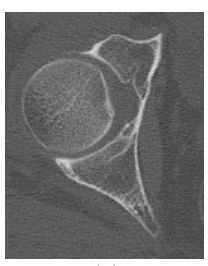

(c)

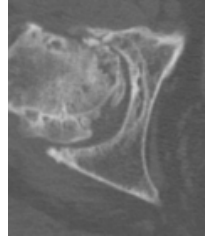

(i)

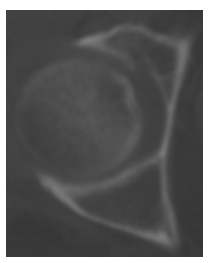

(d)

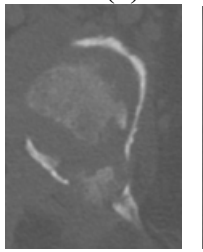

(j)

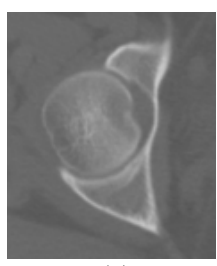

(e)

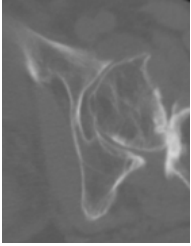

$(\mathrm{k})$

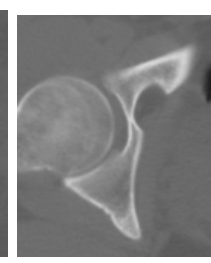

(f)

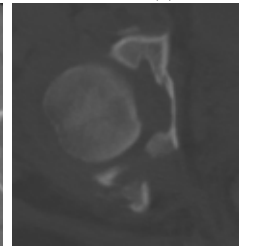

(1)

Fig. 1. Representative samples from the four groups of hip joints. Group \#1 (a c): The acetabulum and the femoral head in the hip are well separated from each other; Group \#2 (d f): The joint space is narrow in several slices; Group \#3 (g i): The acetabalum and the femoral head are adjacent physically, the shape of the femoral head seems to be irregular; Group \#4 (j 1): The joint spaces in many slices of the hip joint are completely disappeared or the acetabalum is very irregular.

set ranges from 85 to 217 . The resolution of every slice image is $512 \times 512$. According to literature [16], these CT data-sets are grouped as follows:

Group \#1: The acetabulum and the femoral head in the hip are well separated from each other, the distribution of bone intensity throughout the hip is even, and the gray level of bone tissues is considerably higher than that of the surrounding tissues. In Figures 1(a)-1(c) three typical slices of this group are shown.

Group \#2: The joint space is narrow in several slices of each data-set, i.e. the acetabulum and the femoral head are close to each other, both cortical and spongy bone tissues have higher image intensity than their surrounding tissues. In Figures 1(d)-1(f) three typical slices of this group are shown.

Group \#3: The acetabulum and the femoral head are adjacent physically, the shape of the femoral head seems to be irregular due to the pathology and malformation of the pelvis and the femur, and the bone tissues in different parts of the acetabulum and the femoral head are not uniformly distributed. In Figures 1(g)-1(i) three typical slices of this group are shown.

Group \#4: The joint spaces in many slices of the hip joint completely disappear or there are breakages in the hip bones due to the severity of a bone disease, malformation or damnification. In Figures 1(j)-1(1) three typical slices of this group are shown.

\subsection{Edge point detection}

Edge detection always is paid enough attention to in segmentation of hip joints [4,7,16-18] because edge points are the basic tool to separate different tissues. Cheng et al.'s [8] combination of valleyemphasized images (V-E image) and the Bayes decision rules can be also considered as an edge detection method because it can binarize the bone tissue and non-bone tissues in CT images. For convenience, it is called as valley-Bayes (V-B) detector whose working flow is shown in Figure 2. Note that the image obtained by the detector is a binary image and is called the valley-Bayes (V-B) image in this paper. Figure 3 gives a V-B image.

Because the V-B detector can obtain more accurate edge points than other edge detectors for hip joints [8], it is used in this paper. However, the surface of acetabulum can not be obtained only by the 


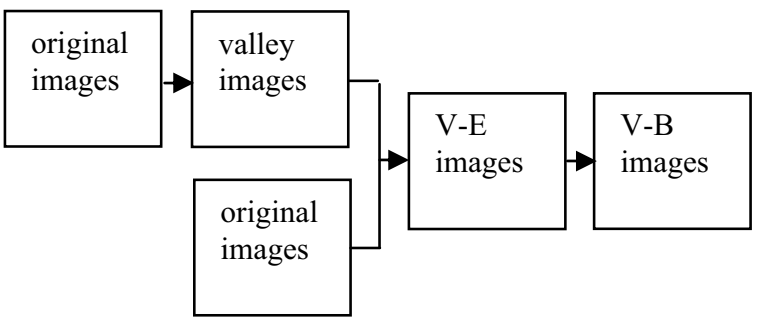

Fig. 2. Flow to obtain the V-B Image.

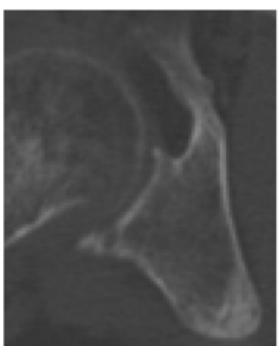

a

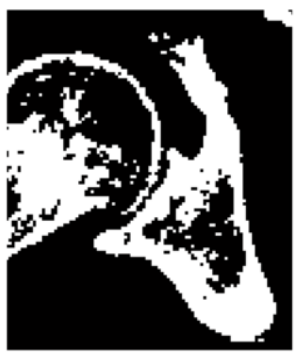

b
Fig. 3. An example of V-B image. (a) original image; (b) V-B Image.

V-B detector because there are many holes, gaps and noises (see Figures 3(b) and 4(b)). Cheng et al. avoid the disadvantage by filling holes and removing noise regions. The drawback of the repairing method is that sizes of the filling diamond and noise region have to be manually given, which largely effects the accuracy of the segmentation. Furthermore, the method also can't assure that these holes are close. See Figure 1(d), there are also probably bone fragments and serious malformations. In these cases, to fill holes is probably a dilemma. So, tasks to fill holes and remove noises should be avoided. The work of this paper is to separate acetabulums and femurs using surfacing fitting method in order to avoid such tasks.

\subsection{Normal vector computation}

Our surfacing fitting method has to use normal vectors of edge points. We use the follwing method to compute normal vectors for edge points in the V-B image:

1. Compute $\boldsymbol{N}_{1}=\nabla \boldsymbol{I}$

2. Using the origin of coordinates as the symmetry point, overturn $I$ and compute $\boldsymbol{N}_{2}=\nabla \boldsymbol{I}$

3. $\boldsymbol{N}=\left(\boldsymbol{N}_{2}-\boldsymbol{N}_{1}\right) /\left|\boldsymbol{N}_{2}-\boldsymbol{N}_{1}\right|$, where $0 / 0=0$.

Since the V-B image is a binary image, it is not necessary to use a Gauss smoothness process for the image to compute normal vectors. Figure 4 gives the example to compute normal-vectors.

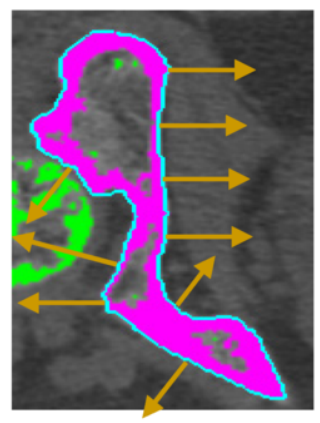

(a)

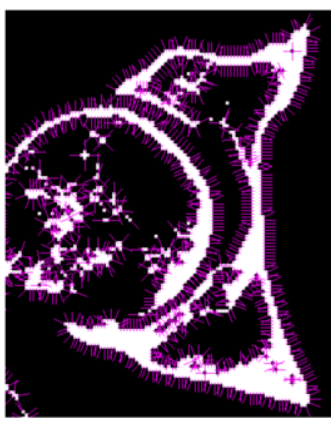

(b)

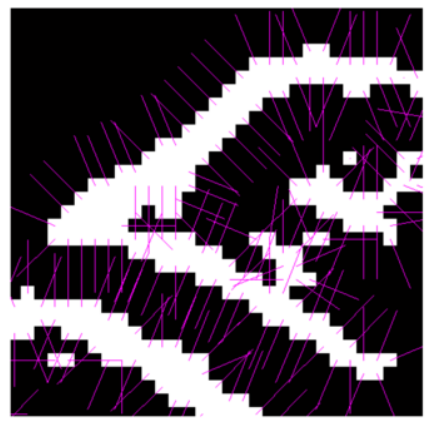

(c)

Fig. 4. Example to compute normal vectors. (a) normal vectors of boundary should point to outer side; (b) normal vectors computed by the method in this paper; (c) local magnification of a region. 


\subsection{Optimum surface fitting for acetabulum}

Zoroof et al. [16] give a rough ellipsoid fitting method for the femur head. Its assumptions are as following:

1. the center of the femur is on the slice(called the main slice) on which the top of the greater trochanter is, as shown in Figures 5(a) and 5(b). For convenience, denote the slice as $\Pi_{o}$;

2. pixels respectively in the femur and the acetabulum in the slice should be separated, as shown in Figure 5(c);

3. for the convex-hull formed by pixels in the femur, it has a center. Link the center and the top of the greater trochanter and form a line segment. The middle point of the line segment should be about the boundary of the femur head, as shown in Figure 5(d);

4. the shape of the contour of the femur head is close to a circle, denote it as $\Gamma$;

5. the 3D shape of the femur head can be approximately described by an ellipsoid whose equation is Eq. (1) in this paper.

$$
\left(x-x_{0}\right)^{2} / a^{2}+\left(y-y_{0}\right)^{2} / a^{2}+\left(z-z_{0}\right)^{2} / b^{2}=1
$$

6. in Eq. (1), the value of a is set as the radius of the circle $\Gamma$. the value of $2 b$ is set as the sum of all distances between every two slices. All these referred slices contain the femur head.

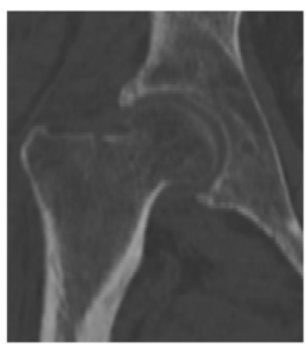

(a)

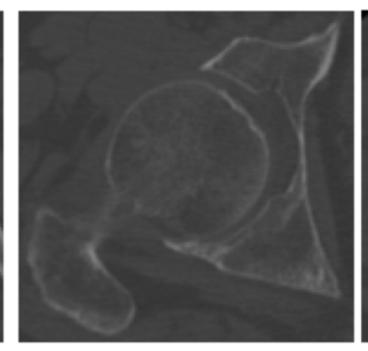

(b)

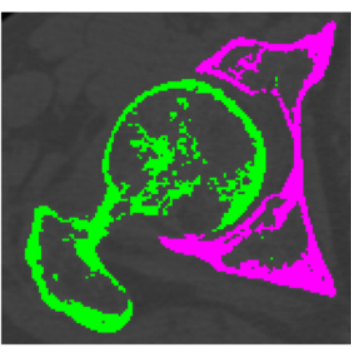

(c)

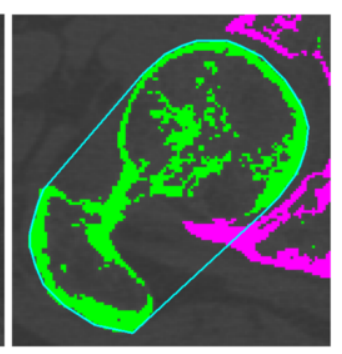

(d)

Fig. 5. Zoroof et al.'s process to obtain the center of the femur head: (a) select a reconstructed coronal image with the greater trochanter of the segmented femur. It is considered that center of the femur head is on the slice that passes through the top of the greater trochanter (called as the main slice). (b) pick the main slice; (c) assume that there has been a method by which the femur and hip bone can be separated in the main slice; (d) calculate the convex-hull(denoted by the cyan polygon) of the femur region. Consider that the center of the convex-hull is the center of the femur head.

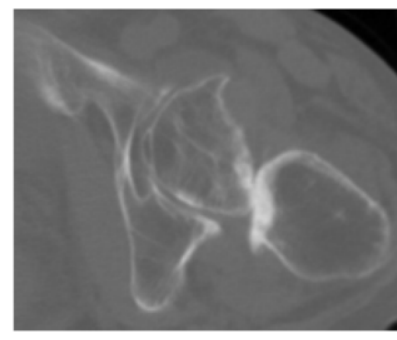

(a)

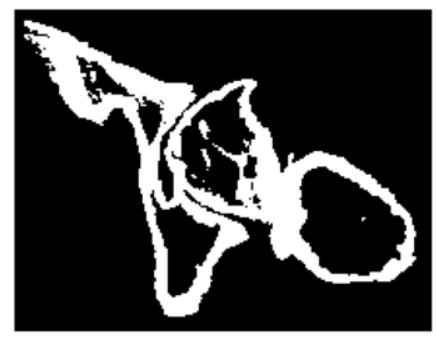

(b)

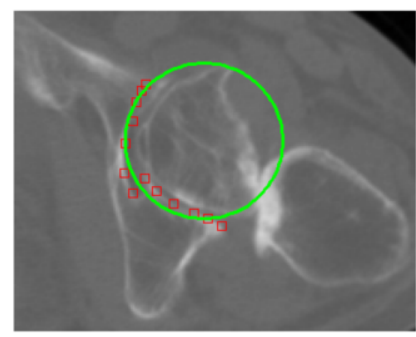

(c)

Fig. 6. An example that the femur and hip bone are connective in the main slice no matter how we enhance and heuristically segment the image. In this case, the method in Figure 7 can't be used to determine the center of the femur head. (a) the original image of the main slice; (b) The V-B image; (c) fitting circle using picked points. 
In fact, the above assumption (ii) is not correct for $\Pi_{o}$ in some cases, especially in Group \#3 and Group \#4, because pixels in the femur head and the acetabulum are always connected in some cases no matter how we enhance the image in the slice, as shown in Figures 6(a) and 6(b). Consequently, instead of using the assumption (ii) and (iii), we pick several points of the acetabulum in the slice $\Pi_{o}$ since the slice has to be manually located, as shown in Figure 6(c). These picked points are used to fit a circle. By using other assumptions, an ellipsoid $S^{0}$ can be obtained. However, $S^{0}$ is not an accurate ellipsoid because of the following two facts:

1. The ellipsoid only uses lengths of two axes. That is, the degree of freedom of the ellipsoid is low.

2. methods to evaluate ellipsoid center and lengths of two axes are rough: the center is manually evaluated; the circle is fitted by picked points.

Consequently, we improve the accuracy of the ellipse $S^{0}$ using an iterative process. $S^{0}$ is the starting quadric surface of the iterative process. In sub-section 2.2 and sub-section 2.3, an edge point set $E$ and their normal vectors are obtained. Assume that a point set $P^{0}$ can be obtained from $E$ using $S^{0}$. Using $P^{0}$ and a fitting method, a surface $S^{1}$ can be obtained. A point set $P^{i}$ can be obtained from $E$ using $S^{i}$. So, an iterative process appears as shown in Figure 7.

In our implementation, the fitting error is investigated. When the fitting error doesn't decrease, the iterative process stops. Now we give the reason that the iterative process in Figure 7 is reasonable. Note that Zoroof et al. gives a method to capture edge points of femur heads according to a rough contour. The method is also an iterative process based on the minimum distance matching principle after the image is binarized. It investigates the section curves of $S^{0}$ in every slice and calls them as the rough boundaries. A point in a rough boundary is called a boundary point. The process to refine a rough boundary can be generalized as following:

Step 1. Smooth the rough boundry (Simplified as "Smooth");

Step 2. Compute the normal line for every boundary (Simplified as "Normal");

Step 3. Adjust a circle around the boundary point (Simplified as "Mask");

Step 4. Find the minimum and maximum gray values image inside the circular mask;

Step 5. Binarize the gray image for a range of thresholds based on the above minimum and maximum values.

Step 6. Find the optimum threshold value by calculating the variances of bone tissue regions and non-bone tissue regions from the image pixels and then by selecting a threshold value that minimizes the total variances.

Step 7. Replace the current boundary value with the closest bone tissue point (Simplified as "Capture");

Step 8. Go to Step 1 if the number of desired iterations is not satisfactory, otherwise stop (Simplified as "Result").

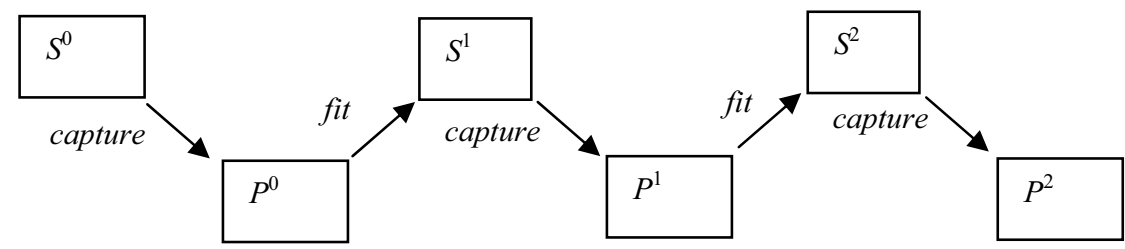

Fig. 7. The iterative process to obtain the optimization surface. 


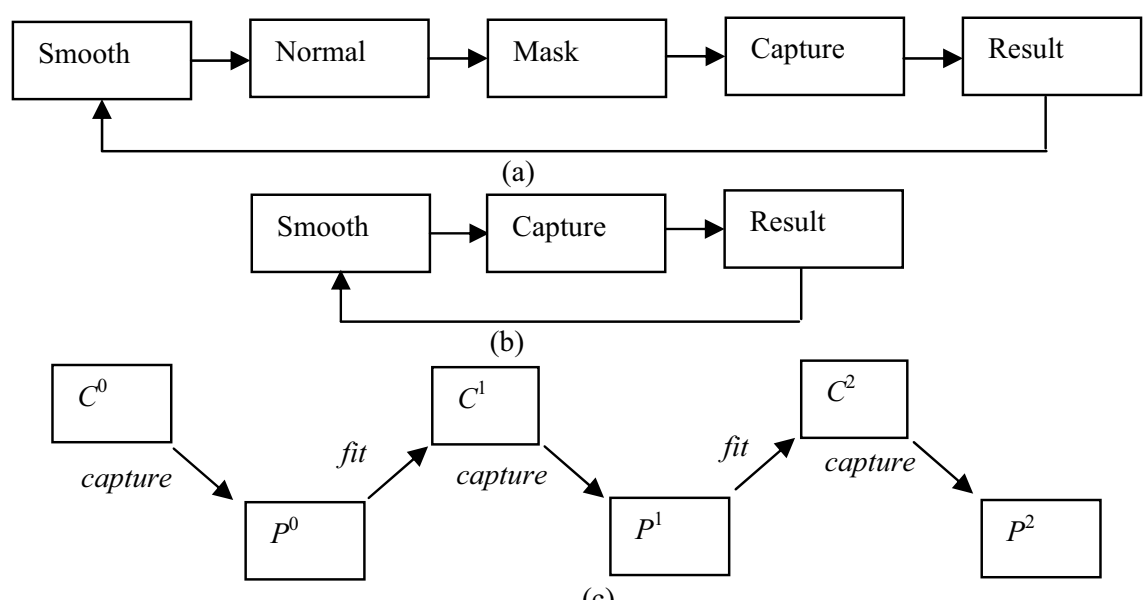

(c)

Fig. 8. Analyze Zoroof et al.'s refining process. (a) the flow after overlooking binarization steps; (b) the flow after overlooking two necessary steps in the flow in (a); (c) re-written form of Zoroof et al.'s refining process.

Investigate the above steps 4-6. The 3 steps form a local binarization technique. The aim is to recognize bone tissues as accurate as possible. Because the Bayes rule (see subsection 2.2) is a more accurate technique to recognize bone tissues around boundaries of bones and the V-B image has been used in this paper, steps 4-6 can be left out for the refine process. So, the process can be simplified as the flow in Figure 8(a).

If consider that "compute normal line" is necessary, it can be unwritten in the flow in the Figure 8(a). Because steps 4-6 have been left out, the function of the mask is only to give the search range, which can be replaced by setting a distance parameter $\delta$. So, the "Mask" can be unwritten. Consequently, the flow in Figure 8(a) can be simplified as the flow in Figure 8(b). Assume that the curve obtained by linking captured points and the smooth step be $C^{i}$. The captured point set is $P^{i}$. The flow in Figure 8(b) can be rewritten as the form in Figure 8(c). According to Figures 7 and 8(c), it can be found that the two flows are almost same. However, there is a large difference between our process and Zoroof et al.'s process: Zoroof et al. enlarges the flexibility of the rough boundary to infinity and the infinite flexibility curve is used in every iterative step, which is an important cause to capture error edge points. In our process, we enlarge flexibility from two-freedom to multi-freedom. The advantage to use flexibility-fixed surfaces in the iterative phase is that the fitted surfaces in next iterative step will go away these error points instead of interpolating them when error captured points appear. Another important difference between our process and Zoroof et al.'s process is that Zoroof et al. capture edge points only using a distance match(see "capture" step in Zoroof et al.' process) while we use the distance match and the normal match, which will be discussed in subdection 2.8.

\subsection{Fitting acetabulum using quadric surfaces}

Assume that there have already been some points of the acetabulum. Fitting methods for acetabulum (or femur head) have been researched in some literatures. The roughest method is the sphere fitting [17]. Peng et al. [15] and Zoroof et al. [16] report that a two-freedom ellipsoid denoted by Eq. (1) is acceptable. Though the ellipsoid fitting method has a good fitting accuracy [16], it is not reasonable to consider that the axes of the ellipsoid are parallel to coordinate axes, which is noticed by $\mathrm{Hu}$ et al. [23]. They take the following ellipsoid: 


$$
\left(x-x_{0}\right)^{2} / a^{2}+\left(y-y_{0}\right)^{2} / b^{2}+\left(z-z_{0}\right)^{2} / c^{2}=1
$$

By using rotation parameters and rotation matrices, they make axes of ellipsoid be able to be in arbitrary direction. These parameters are solved by a non-linear optimization model. The method is strict. However, to solve the optimization model is time-consuming. Furthermore, the process of solution is probably diverging [24]. We design two-phase method to compute the ellipsoid: (1) fit the acetabulum by using a sphere; (2) fix the center and fit surface by using the following equation:

$$
A x^{2}+B y^{2}+C z^{2}+D x y+E y z+F z x+G x+H y+I z+J=0
$$

In our implementation, we translate the sphere center to the origin of coordinates. In this case, Eq. (3) is rewritten as follows:

$$
A x^{2}+B y^{2}+C z^{2}+D x y+E y z+F z x+J=0
$$

For convenience, we give the following concepts by using Eq. (4):

- two DOF(degree of freedom) fitting: $A=B, D=E=F=0$, which is equivalent to Eq. (1)

- three DOF fitting: $D=E=F=0$, which is equivalent to Eq. (2)

- multi-DOF fitting: $A, B, C, D, E, F, J$ are arbitrary, which is equivalent to the method in [23]. Our computation can be converted into a least square computation. A comparison between different fitting methods is shown in Figure 9.

\subsection{Acetabulum fringe plane determination}

In the iterative process in Figure 7, we have to collect matched edge points for iterative surfaces. However, an ellipsoid is close, which results in edges points in femur heads is collected. In order to avoid such a disadvantage, we construct the fringe plane $\Sigma$ for acetabulum. According the anatomy structure of the acetabulum, there should be an acetabulum axis and an acetabulum center (see Figure 10 ). So, a plane $\Sigma$ can be determined by the axis and the center. All points (form the set $P$ ) in the acetabulum should be on a side of the plane $\Sigma$ because the acetabulum is on a side of the plane $\Sigma$ and $P$ is

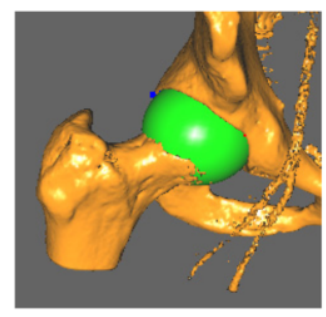

(a)

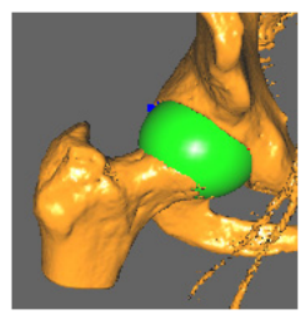

(b)

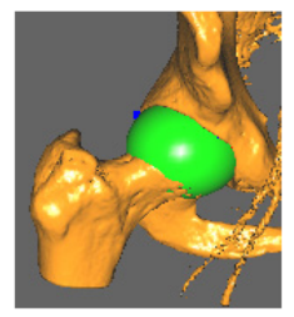

(c)

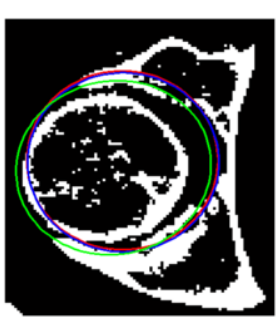

(d)

Fig. 9. acetabulum fitting methods (a) sphere fitting(average error: $2.3595 \mathrm{~mm}$ ); (b) two DOF fittings (average error: $2.3255 \mathrm{~mm}$ ); (c)multi-DOF fittings (average error: $2.0189 \mathrm{~mm}$ ); (d) accuracy comparison of different fitting method in a slice. red: sphere fitting method; blue: two DOF fitting method; green: multi-DOF fitting method. 
a point set in the acetabulum. The side is called as the $\Sigma$ positive side. Another side is called the $\Sigma$ negative side. According to anatomy properties of the acetabulum, the angle (inclination angle) between the projection of the acetabulum axis on the sagittal plane and the $z$ coordinate axis is the about 45 degree. The angle (anteversion angle) between the acetabulum axis and the sagittal plane is about 15 degree, as shown in Figure 10. According to Figure 10, for left acetabulum, the angle between the acetabulum axis and $x$ axis is 75 degree; for right acetabulum, the angle between the acetabulum axis and $x$ axis is 135 degree. So, an acetabulum axis direction can be expressed as:

$$
\boldsymbol{n}=(\cos \theta \cos \varphi, \sin \theta \cos \varphi, \sin \varphi)
$$

When the iterative process in the sub-section 2.4 ends, we assume that the optimization surface capture a point set $P$. So, an optimization model can be obtained:

$$
\max \sum_{i=0}^{N_{p}}\left|\left(\boldsymbol{p}_{i}-\boldsymbol{o}\right) \boldsymbol{n}\right|^{2}
$$

Where $\boldsymbol{p}_{i} \in P$ and $\boldsymbol{n}$ is expressed by Eq. (5), $\boldsymbol{o}$ is the sphere center of in the two-step fitting method. Using the optimization model, an accurate acetabulum axis direction can be obtained. Using the last iteration ellipsoid and the direction, the last capturing points can be obtained

\subsection{Edge point capture}

For a set of CT images, the slice spacing is usually larger than the pixel spacing, which probably results in un-connection of pixels of acetabulum in two adjacent slices. So, our method automatically captures points slice by slice. For the $k$-th slice, we can know that an ellipse that is an intersection curve between the fitting ellipsoid and the slice; a line that is an intersection line curve between the acetabulum fringe plane and the slice; a center that is the projection of the ellipsoid center to the slice; a directed line which is the projection of the ellipsoid axis to the slice, as shown in Figure 11(a). Using this information, we can obtain a semi-ellipse and its normal vectors, as shown in Figure 11(b). The semi-ellipse is called as the rough contour. Since the normal vectors and edge points have been computed, we give two conditions to make the curve capture edge points. Firstly assume that $\boldsymbol{p}$ is an edge point and $\boldsymbol{q}$ is the closest point in the semi-ellipse to the point, give the following two conditions:

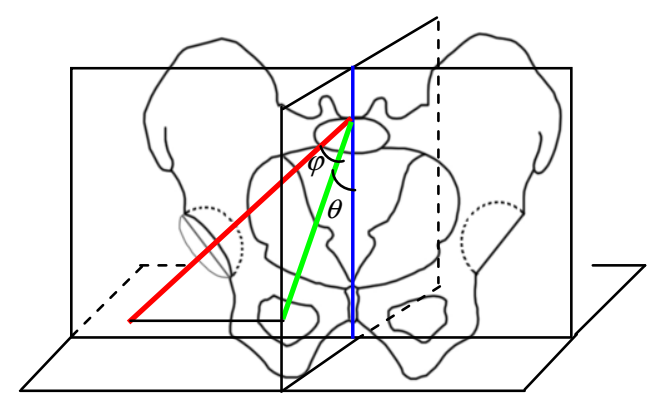

Fig. 10. The anatomy structure of an acetabulum. $\theta$ : anteversion angle; $\varphi$ : inclination angle. 


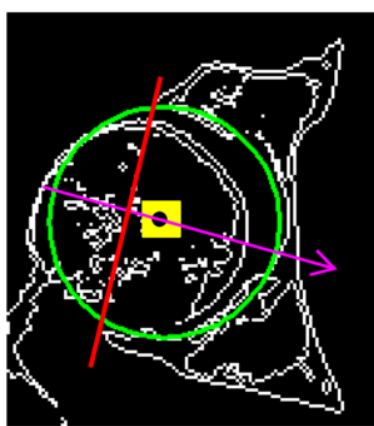

(a)

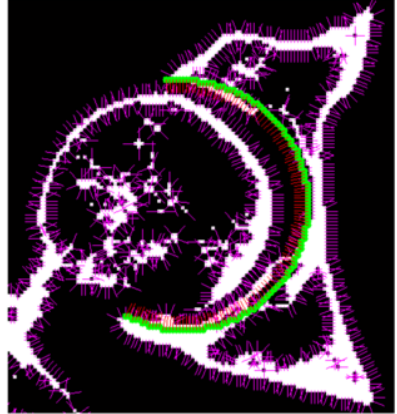

(b)

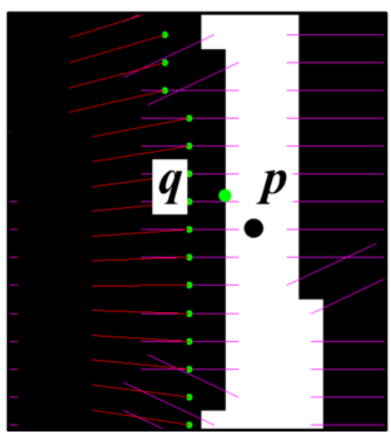

(c)

Fig. 11. Rough contour captures edge points according to distance parameter and normal vector parameter. (a) intersection curve or projection of ellipsoid, acetabulum fringe plane, acetabulum axis and the ellipsoid center; (b) rough contour(green), edge points and normal vectors. (c) a rough contour point $\boldsymbol{q}$ (green point)and its captured point $\boldsymbol{p}$.

(1) $|\boldsymbol{p q}| \leq \delta$

(2) $\boldsymbol{n}_{p} \boldsymbol{n}_{q} \geq \sigma$

For convenience, the above two conditions are called as the initial matching conditions that are respectively called as the distance matching condition and the normal-vector matching condition. When the initial matching conditions can be satisfied, we call that $\boldsymbol{p}$ can be captured by the semi-ellipse. So, it can be captured by the iterative ellipsoid. The edge point $\boldsymbol{p}$ is called is a captured point of $\boldsymbol{q}$. The above two conditions are uniformly called capturing conditions. $\delta$ is a distance threshold value. According to Zoroof et al.'s statistics data, the maximum space between the acetabulums and the femur heads is $d=8^{*} 0.68$. It is an anatomy statistics datum. Assume that the used pixel space is $s$, the $\delta$ is set as:

$$
\delta=d / s
$$

For a set of CT images, $s$ is a constant in any slice. $d$ can use the given value $(8 * 0.68)$ and also be set by an experienced user. $\sigma$ is an angle cosine parameter. In our implementation, it is set $0 . q$ and its captured points is shown in Figure 11(c). Using the capturing method, a set $P$ can be obtained. Obviously, there are some incorrectly recognized points in $P$. There are also some acetabulum edge points that are unrecognized (see Figure 12(a)). Now we have to remove these incorrectly recognized points and complement these unrecognized points. The task is completed by an optimum region selection method. That is, when a normal line of a point of the rough contour intersects several regions, the largest region is retained and smaller regions are removed. The principles of the method are: (1) a point in the acetabulum has a unique matched point in the rough contour along the normal line; (2) regions formed the largest regions and other regions are fragmentary regions about the rough contour. (3) there are usually a path between two edge points of the acetabulum. Any point in the path is an edge point of the acetabulum. Based on the three principles, we use the following process to obtain points of the acetabulum:

(1) Use a region growth method to partition the set $P$, as shown in Figure 12(b). So it is clear that which regions are fragmentary regions and which regions are large regions. The step is designed according to the second principle. Assume that the region set be: 


$$
\mathrm{R}=\left\{R_{1}, \cdots, R_{n}\right\}
$$

Obviously,

$$
\bigcup_{i=1}^{n} R_{i}=P
$$

Every region has only a pixel width (see Figures $12(\mathrm{a})$ and 12(b)). That is, a region is a dicrete curve. So, a region $R_{i}$ has at least an end point. If a region has only an edge point, it has only an end point. Otherwise, a region has two edge points. A region end point is denoted as $\boldsymbol{p}_{i, s}, i$ is the region index and $s$ (equal to 1 or 2 ) is the end point index.

(2) Unite fragmentary regions as best as we can lest regions in the acetabulum are incorrectly removed as fragmentary regions. We collect end pairs that form a set:

$$
\mathrm{P}=\left\{\left(\boldsymbol{p}_{i, s}, \boldsymbol{p}_{j, t}\right) \mid i \neq j, s=1 \text { or } 2, t=1 \text { or } 2\right\}
$$

Eq. (7) shows two end points in an end pair are not in the same region. For any end pair $\left(\boldsymbol{p}_{i, s}, \boldsymbol{p}_{j, t}\right)$ and let

$$
\hat{\mathrm{E}}=\mathrm{E}-\mathrm{P}+\left\{\mathbf{p}_{\mathrm{i}, \mathrm{s}}, \mathbf{p}_{\mathrm{j}, \mathrm{t}}\right\},
$$

we use a graph-based searching process to search paths in $\hat{E}$. Note that every point in $\hat{E}$ has a normal vector that is inherited from $E$. A reasonable path $\Gamma$ should satisfy the following conditions:

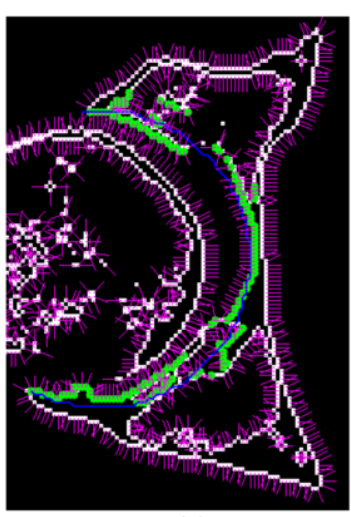

(a)

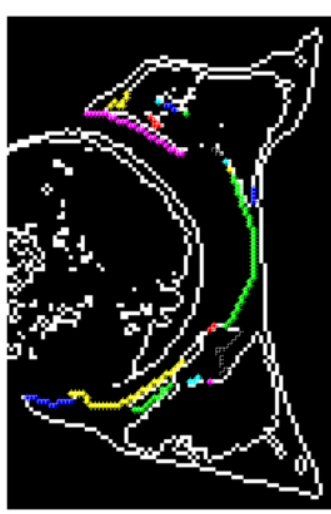

(b)

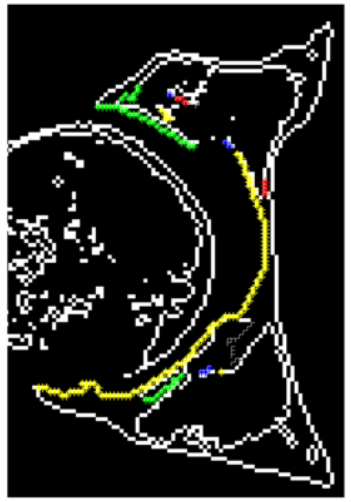

(c)

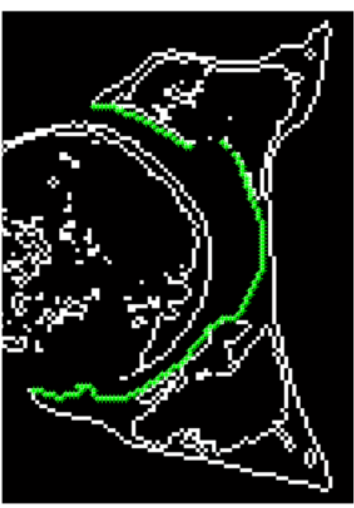

(d)

Fig. 12. A process to capture edge points for the rough contour. (a) captured edge points according to the distance matching condition and the normal-vector matching condition; (b) partition captured edge points into different regions using region growth processes. (c) uniting some regions using graph-based searching processes. (d) remove fragmentary regions using a normal projection technique. 
- Assume that $\boldsymbol{p}_{i, s}$ and $\boldsymbol{p}_{j, t}$ are respectively captured points of $\boldsymbol{q}_{i, s}$ and $\boldsymbol{q}_{j, t}$. The arc length of the ellipse between $\boldsymbol{q}_{i, s}$ and $\boldsymbol{q}_{j, t}$ is $L$. The path between $\boldsymbol{p}_{i, s}$ and $\boldsymbol{p}_{j, t}$ should not larger than $c L$, where $c$ is the enlarging coefficient. In our implement, $c$ is set as 1.2.

- Assume that $\boldsymbol{p}_{1}, \boldsymbol{p}_{2}$ be two points in the path $\Gamma$. Referring to properties of the circle, if the angle between $\boldsymbol{o} \boldsymbol{p}_{1}$ and $\boldsymbol{o} \boldsymbol{p}_{2}$ is less than 90, the angle between $\boldsymbol{n}_{p 1}$ and $\boldsymbol{n}_{p 2}$ is less than 90 degree. In our application, we consider that the angle between $\boldsymbol{n}_{p 1}$ and $\boldsymbol{n}_{p 2}$ is less than 90 if the angle between $\boldsymbol{o p}_{1}$ and $\boldsymbol{o p}{ }_{2}$ is less than 45 degree because of inaccuracy of evaluated normal vectors. If there are several reasonable paths, we select the path with the minimum length. After uniting fragmentary regions, obtained region are shown in Figure 12(c). After the region uniting process, we denote the new region set as:

$$
\mathrm{R}^{\prime}=\left\{R_{1}^{\prime}, \cdots, R_{k}^{\prime}\right\}
$$

(3) For any point in $R_{j}^{\prime}(j=1, \cdots, k)$, assume that it be the captured point of $\boldsymbol{q}$. If the normal line of $\boldsymbol{q}$ intersects several regions, the largest region is retained and smaller regions are removed from $\mathrm{R}^{\prime}$. After smaller regions are removed, the points in retained regions are captured points by the rough contour. After the last iteration step end, captured points form the refined contour (see Figure 12(d)).

It should be pointed out that every captured point can be parameterized on the semi-ellipse according to our captured method. So, these captured points can be sorted according to their parameter. By linking these sorted points, an acetabulum contour forms.

\section{Results and analysis}

Our segmentation method is integrated into our developed system DataSet that is a CT image processing system. When data in this section are tested, the system is running in a PC with $3.40 \mathrm{GHz}$ Intel processor and $1.95 \mathrm{G}$ RAM.

\subsection{Acetabulum fitting analysis}

Select 20 hip joints respectively from 4 groups (see Section 2.1) and fit acetabulum surface using methods in Section 2.5. These fitted points are interactively picked in the interface of our developed system DataSet. In a comparison case, different fitting methods use the same picked points. The aim of such experiments is to test the effectiveness of our multi-DOF method. Table 1 compares fitting errors for several fitting methods. The fitting error is computed using the following equation:

$$
\varepsilon=\sum_{i=1}^{n} d\left(S, \boldsymbol{p}_{i}\right) / n
$$

Where, $S$ is the fitting surface, $\boldsymbol{p}_{i}$ is the fitted point, $d\left(S, \boldsymbol{p}_{i}\right)$ is the Euclidian distance between $S$ and $\boldsymbol{p}_{i}$. From Table 1, we can find that fitting errors become less from sphere fitting to multi-DOF fitting. For any fitting method, fitting errors become larger from Group \#1 to Group \#4, which shows Group \#4 probably has lower segmentation success rate. Figure 13 gives a comparison for different fitting 
Table 1

Compare Several Fitting methods

\begin{tabular}{|l|l|l|l|l|l|l|}
\hline \multirow{2}{*}{ Group } & \multirow{2}{*}{$\begin{array}{l}\text { Case Num- } \\
\text { ber }\end{array}$} & \multirow{2}{*}{$\begin{array}{l}\text { Point } \\
\text { Number }\end{array}$} & \multicolumn{2}{l|}{ Fitting Methods $(\mathrm{mm})$} \\
\cline { 4 - 7 } & & Sphere & 2 DOF & 3 DOF & Multi-DOF \\
\hline$\# 1$ & 20 & 30 & 2.4657 & 2.4423 & 2.4326 & 2.0296 \\
\hline$\# 2$ & 20 & 30 & 2.4683 & 2.4547 & 2.4568 & 2.0873 \\
\hline$\# 3$ & 20 & 30 & 2.5667 & 2.5177 & 2.5103 & 2.3426 \\
\hline$\# 4$ & 19 & 30 & 2.9527 & 2.9235 & 2.9146 & 2.7576 \\
\hline Total & 79 & 30 & 2.6134 & 2.5846 & 2.5786 & 2.3043 \\
\hline
\end{tabular}

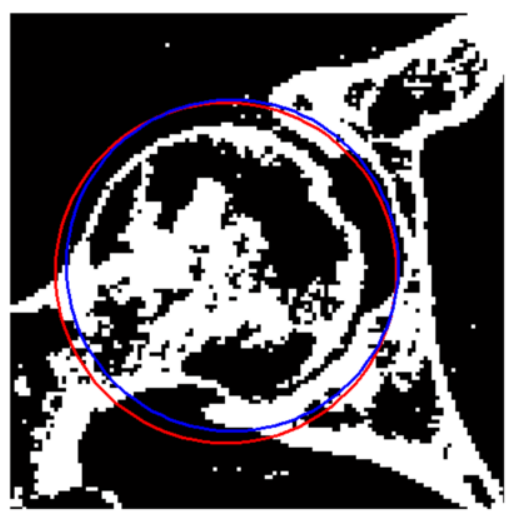

(a)

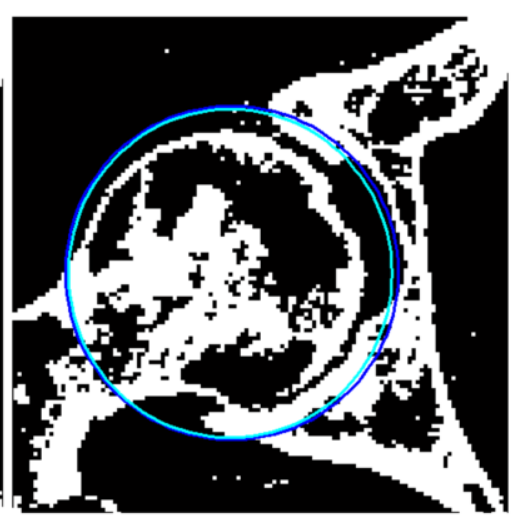

(b)

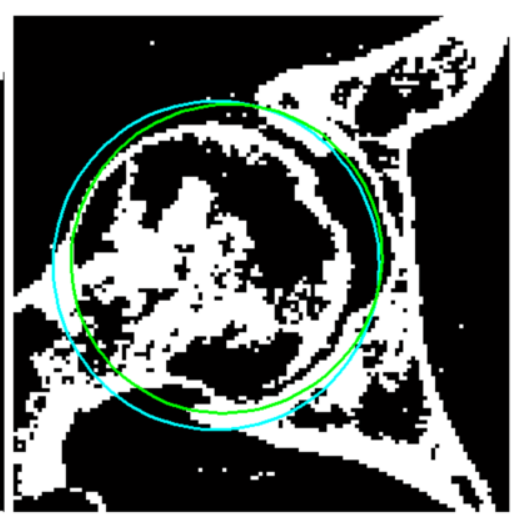

(c)

Fig. 13. A comparison for different fitting methods in an example. (a) sphere fitting(red) and two-DOF fitting(blue); (b) twoDOF fitting (blue) and three-DOF fitting(cyan); (c) three-DOF fitting (cyan) and multi-DOF fitting(green).

methods in an example. In the Figure 13, it can be found that there are large differences between sphere fitting and two-DOF fitting, between 3-DOF fitting and multi-DOF fitting. However, there seems be small difference between 2-DOF fitting and 3-DOF fitting. Such a comparison is the cause that we select the multi-DOF fitting.

\subsection{Iterative process analysis}

Our automatic segmentation method needs an initial ellipsiod. Using the initial iterative ellipsoid, our method can segment the acetabulum without manual interventions. The initial ellipsoid is obtained by two interactions and an evaluated parameter: (1) select the main slice on which the center of the acetabulum is; (2) pick several acetabulum points on the main slice. The evaluated parameter is the number of slices containing the acetabulum. Since these parameters are obtained by interactions and evaluations, the accuracy of the ellipsoid is low. Our iterative processes improve the accuracy for the ellipsoid. Figure 14 gives a contour-changing sequence in a slice for the ellipsoid iterative process. Figure 15 gives the relation curves between captures point number and iteration step number, between errors and iteration step number. 


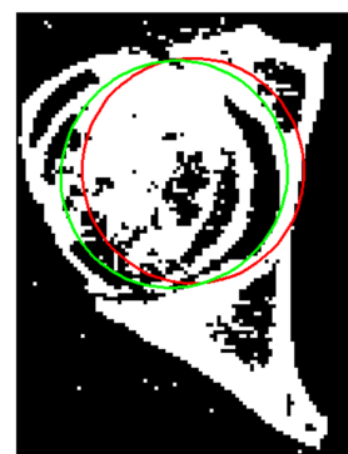

(a)

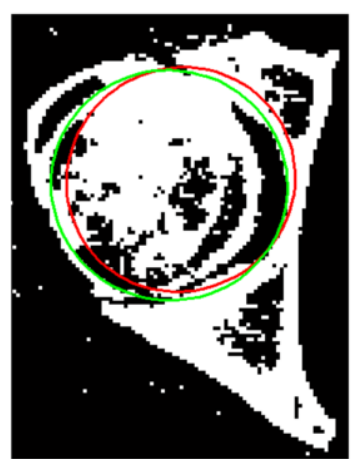

(b)

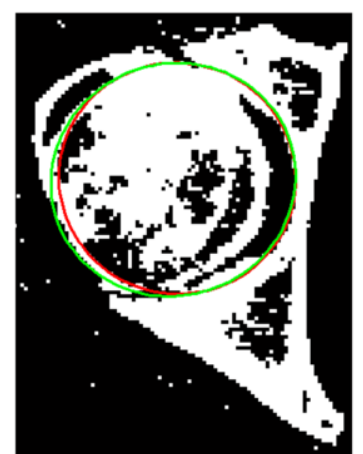

(c)

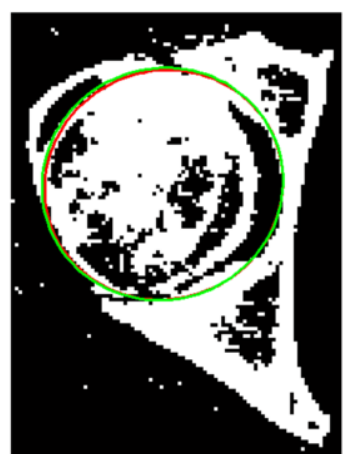

(d)

Fig. 14. Contour-changing sequence in a slice for the ellipsoid iterative process. (a) $1^{\text {st }}$ iteration; (b) $2^{\text {nd }}$ iteration; (c) $3^{\text {rd }}$ iteration; (d) $4^{\text {th }}$ iteration. Red: contour in previous iterative step; green: contour in current iterative step. In (a), the red contour is the initial contour.

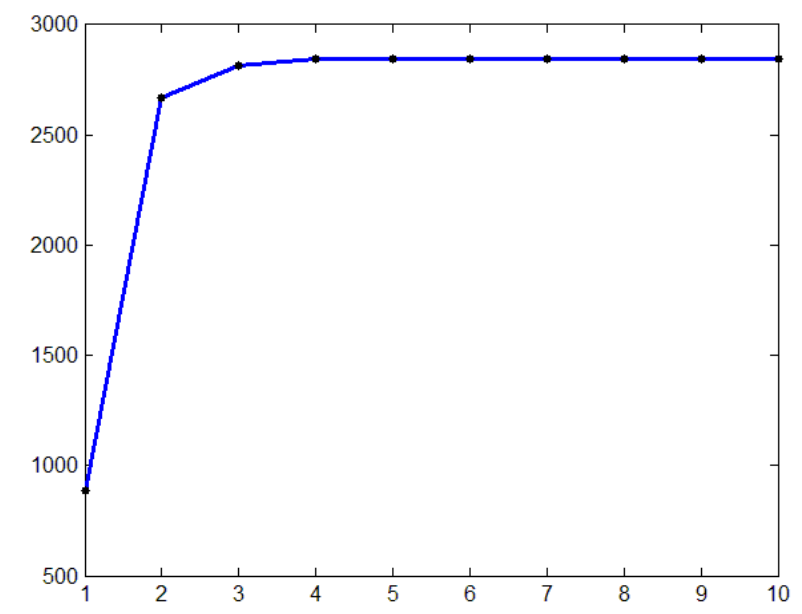

(a)

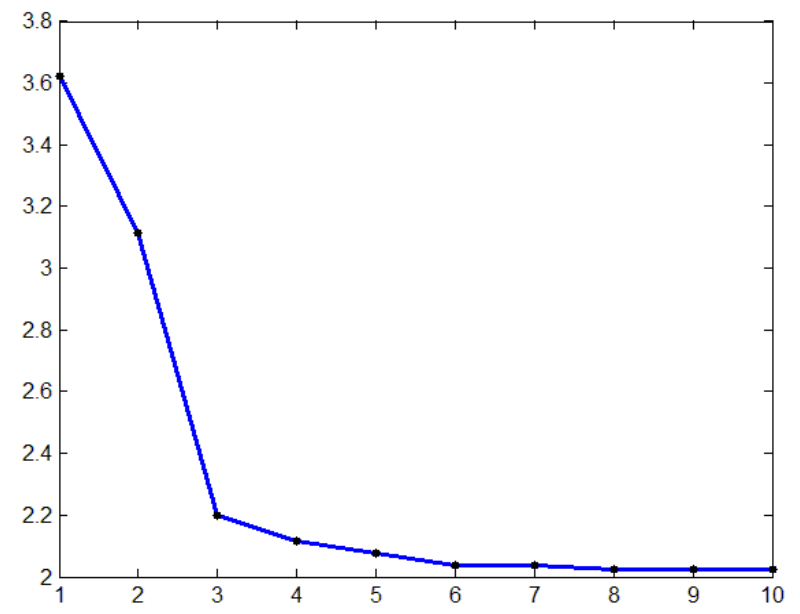

(b)

Fig. 15. Parameters change in the iterative process. (a) relation curve between captures point number and iteration step number; (b) relation curve between error and iteration step number.

\subsection{Consuming time analysis}

Assume that our comparisons start from the V-B image for which a boundary point re-collection process is used. We use such a process only because it is a necessary step for Cheng et al.' work [8]. See Figure 16. A 6-voxel neighborhood structure is used for a voxel of the bone tissue (black). If there is a non-bone tissue voxel among 6 adjacent pixels, the voxel (black) is a boundary point of the bone tissue.

Table 2 gives a consuming time statistics to separate the acetabulum. From Table 2, the procedure to capture edge points consumes most of the time because every edge point is investigated whether a point is captured by the previous ellipsoid and region growth methods have to be repeatedly used. From Table 3, it can be found that the time consume focuses on repairing holes and removing noises because region growth methods have to be repeatedly used to recognize hole regions and noise regions. 


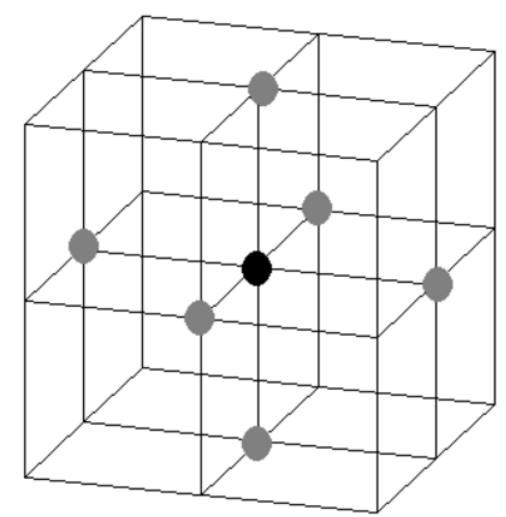

Fig. 16. 3D neighbourhood structure of voxel $\boldsymbol{x}$ (black point).

Table 2

Required time for our algorithm

\begin{tabular}{|c|c|c|c|c|c|c|c|c|}
\hline \multirow{2}{*}{ group } & \multirow{2}{*}{ image size } & \multirow{2}{*}{$\begin{array}{l}\text { slices } \\
\text { includ- } \\
\text { ing ace- } \\
\text { tabulum }\end{array}$} & \multirow{2}{*}{$\begin{array}{l}\text { time to } \\
\text { extract } \\
\text { edge } \\
\text { points(s) }\end{array}$} & \multirow{2}{*}{$\begin{array}{l}\text { time to } \\
\text { compute } \\
\text { cormals for } \\
\text { edge } \\
\text { points(s) }\end{array}$} & \multicolumn{2}{|c|}{ iteration time (s) } & \multirow{2}{*}{$\begin{array}{l}\text { total } \\
\text { time(s) }\end{array}$} & \multirow{2}{*}{$\begin{array}{l}\text { average } \\
\text { time in a } \\
\text { slice(s) }\end{array}$} \\
\hline & & & & & fit & capture & & \\
\hline$\# 1$ & $512 \times 512 \times 96$ & 21 & 4.0032 & 5.6329 & 2.6831 & 41.2731 & 53.5563 & 2.5503 \\
\hline$\# 2$ & $512 \times 512 \times 97$ & 35 & 7.3251 & 9.6324 & 4.3126 & 63.2651 & 91.5352 & 2.6153 \\
\hline$\# 3$ & $512 \times 512 \times 98$ & 35 & 7.3364 & 9.7361 & 4.2268 & 74.3122 & 95.6115 & 2.7318 \\
\hline$\# 4$ & $512 \times 512 \times 101$ & 37 & 7.6265 & 10.0147 & 4.9532 & 83.6329 & 106.2270 & 2.8710 \\
\hline
\end{tabular}

Table 3

Required time for our algorithm in a slice

\begin{tabular}{|l|l|l|l|l|l|l|l|}
\hline group & image size & $\begin{array}{l}\text { slices } \\
\text { include- } \\
\text { ing ace- } \\
\text { tabulum }\end{array}$ & $\begin{array}{l}\text { time to } \\
\text { repair holes } \\
(\mathrm{s})\end{array}$ & $\begin{array}{l}\text { time to } \\
\text { remove } \\
\text { noises (s) }\end{array}$ & $\begin{array}{l}\text { time to extract edge } \\
\text { points(s) }\end{array}$ & $\begin{array}{l}\text { total } \\
\text { time(s) }\end{array}$ & $\begin{array}{l}\text { average } \\
\text { time in a } \\
\text { slice (s) }\end{array}$ \\
\hline$\# 1$ & $512 \times 512 \times 96$ & 21 & 42.1731 & 16.6233 & 11.8275 & 70.6239 & 3.3630 \\
\hline$\# 2$ & $512 \times 512 \times 97$ & 35 & 77.2256 & 29.3364 & 18.2364 & 124.7984 & 3.5657 \\
\hline$\# 3$ & $512 \times 512 \times 98$ & 35 & 78.8457 & 30.9471 & 20.7183 & 130.5111 & 3.7289 \\
\hline$\# 4$ & $512 \times 512 \times 101$ & 37 & 86.4432 & 35.6255 & 25.3781 & 147.4468 & 3.9850 \\
\hline
\end{tabular}

The cause that our method has smaller time consume is that our region growths are implemented only in edge points while Cheng's region growths [8] are implemented in whole images or interest regions.

The most difficulty to fill holes and remove noises is that there is no a standard that how large a noise region is and how large the gap of an open hole is, which is a root that Cheng's method fail or has segmentation error(see Figures 3(b) , 4(b), 6(b) and 14).

\subsection{Resulting contour analysis}

In order to be convenient for comparison, we use Zoroofi et al.'s evaluation method as following $[8,16]$ : 
(1) For each hip, mean values of manually segmented bone pixels are used as the references and denoted by $P_{\text {ref. }}$.

(2) True Positive $(T P)$, False Positive $(F P)$, and False Negative $(F N)$ pixels a recalculated. These values are corresponding to the following $C \mathrm{~T}$ tissues:

- (a) TP pixels $\left(P_{T P}\right)$ : correctly segmented bone tissues;

- (b) $F P$ pixels $\left(P_{F P}\right)$ : non-bone tissues recognized as bone tissues due to the failure of the technique;

- (c) $F N$ pixels $\left(P_{F N}\right)$ : missed bone tissues.

(3) Success rate of segmentation technique is computed by SUCCESS $=\left(P_{T P} / P_{\text {ref }} \times 100\right.$

(4) Error is determined by $E R R O R=\left(P_{F P} / P_{\text {ref }} \times 100\right.$

(5) Bone segmentation performance is classified into one of the three groups: good, moderate, and poor.

If (SUCCESS $>90 \%)$ and $(E R R O R<10 \%)$, segmentation performance is good. If (SUCCESS $>70 \%$ ) and $(E R R O R<30 \%)$, segmentation performance is moderate. Otherwise segmentation performance is poor.

Table 4 gives the evaluation data for our method. In Table 4, cases in Group \#1 have a high accuracy ratio, the cause for this is that joint spaces in these cases are large. Cases in Group \#2 and Group \#3 also have high accuracy ratios and their accuracy ratios are close, the cause for this is that joint spaces in these cases always exist. In Group \#3, only femur heads have lesion whereas acetabulums don't. Cases in Group \#4 have a low accuracy ratio, the cause for this is that acetabulums have severe malformation. Furthermore, in some slices, joint spaces disappear. Figure 17 gives 4 examples respectively from 4 groups to show segmented acetabulums. In group\#1 group\#4, it can be found that some parts of green contours are warded off by surfaces. These warded green parts are usually moderate pixels. They correspond to missed bone tissues. In group\#3 and group\#4, there are some slices in which contour can't be recognized.

Table 4

Accuracy ratio of Result Contours

\begin{tabular}{|c|c|c|c|c|c|}
\hline \multirow[t]{2}{*}{ Group } & \multirow{2}{*}{$\begin{array}{l}\text { Case } \\
\text { Number }\end{array}$} & \multirow{2}{*}{$\begin{array}{l}\text { Slice } \\
\text { Number }\end{array}$} & \multicolumn{3}{|c|}{ Resulting curves } \\
\hline & & & Good (\%) & Moderate (\%) & Poor (\%) \\
\hline$\# 1$ & 20 & 403 & 78.1 & 19.6 & 2.3 \\
\hline$\# 2$ & 20 & 387 & 72.6 & 24.7 & 2.7 \\
\hline$\# 3$ & 20 & 405 & 65.3 & 26.8 & 7.9 \\
\hline$\# 4$ & 19 & 376 & 50.1 & 20.7 & 29.2 \\
\hline Total & 79 & 1571 & 66.5 & 22.9 & 10.5 \\
\hline
\end{tabular}

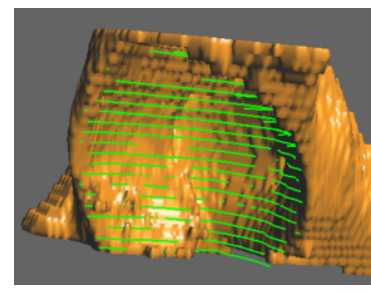

(a)

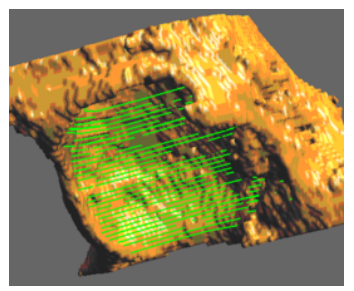

(b)

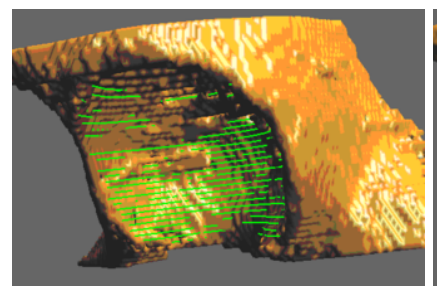

(c)

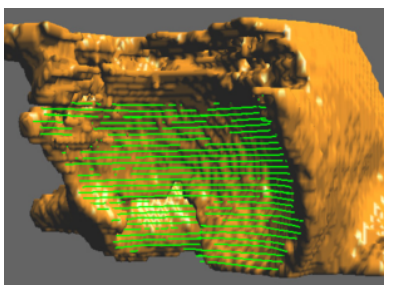

(d)

Fig. 17. Examples for Automatic Segmentation. (a) Group\#1; (b) Group\#2; (c) Group\#3; (d) Group\#4. Green Contours: Contours extracted by our algorithm. 


\section{Discussion}

As shown in Table 4, the algorithm in this paper is effective to achieve the result from being moderate to good in in $97.7 \%(78.1+19.6), 97.3 \%(72.6+24.7)$ and $92.1 \%(65.3+26.8)$ of cases in Group \#1, Group \#2 and Group \#3, respectively. Such a high right ratio makes our algorithm have a high success ratio to segment cases from Group \#1 to Group \#3. Generally, to accurately segment every slice is difficult even for cases in Group \#1 because there are probably several slices in which joint spaces are blurry, which is shown in Table 4 (the poor ratio is $2.3 \%$ for Group\#1). As shown by experiments, our method has a high accuracy degree even for acetabulums with severe malformation. In Table 5, the effective ratio is $70.8 \%(50.1+20.7)$. In Table 6 , the contour error ratio is $29.2 \%$. That is, most extracted contours are available.

Compared with similar methods, the accuracy of our method is largely improved, error ratios $21 \%$, $24 \%, 26 \%, 12.8 \%$ and $10.5 \%$ respectively for the method in $[8,16,21,25]$ and in this paper (see Table 4), which is due to the following three reasons: (1) the quadric surface fitting method has higher fitting accuracy than other shape estimating methods(see Table 1); (2) normal vectors are used as matching information while the information is not used by other methods; (3) our optimum region selection method is actually an intelligentized method to remove noises and fill holes. The method can accurately capture points (see subsection 2.7).

Our method is actually a heuristic method using an ellipsoid as the prior knowledge. There are also other methods using the previous contours as the prior knowledge (also called as heuristic contours) [10-14,26,27]. However, error diffusion makes them suffer from two disadvantages: (1) low automation degree due to low extracted contour accuracy. Djemal et al. [10] reports their method could automatically manage several slices. Liu et al. [27] suggests a hybrid seed contour strategy, e.g., after five slices, users interactively construct the seed contours [27]. (2) manual supervision and modification are necessary in the process of contour extraction. Compared with those methods using the previous contours as the prior knowledge, our method is unsupervised. Since our point-capturing method can accurately capture points according to heuristic contours, it is helpful to consider a combination for our point-capturing method and other heuristic methods.

It has to pointed out that only to use binarized images, such as V-B images, threshold segmentation images, region growth images, isn't enough to obtain boundaries(or called as extract contours) of interesting tissues because such binarized images are obtained using the local information, which makes interesting tissue regions have gaps or are connected with other regions. Cheng et al. [8] can use V-B images extract contours because they try to fill gaps using hole-filling methods and try to separate interesting tissue regions from other tissues using a 6-connnected 3D structure(see Figure 16). Heuristic contours (ellipses, previous contours) are the global information and they can remove disturbances of incorrect local information. So, our work is to make the segmentation process independent of holefilling, noise-removing and region-separating methods. These methods usually need parameters that are manually set.

\section{Conclusion}

This paper presents an effective algorithm to automatically segment acetabulums from hip joints based on V-B images. The algorithm is programmed and integrated into our developed CT image processing system DataSet. 40 sets of in vivo CT data of actual patients (79 hip joints) are used to evaluate the algorithm. Based on several anatomical and imaging criteria, these groups are classified into 4 
groups. Operator-based quantifications are performed to evaluate our algorithm. Experiments show that our algorithm has the highest accuracy ratio: contour pixel accuracy ratio is larger than $90 \%$ for Group\#1 Group\#3, larger than 70\% for Group\#4. Compared with existing methods, the accuracy of extracted contours is largely improved. Cases in Group\#4 have high error ratio, for which important reasons are that joint spaces disappear in $\mathrm{CT}$ images.

Automatic segmentation of hip joints is a challenging work that can be used as reference to segment other joints. This paper only separates the acetabulums from hip joints. Our future work will combine other heuristic contours and our point-capturing method and present a robust algorithm independent of hole-filling, noise-removing and region-separating methods to obtain contours for all hip bones and femurs.

\section{Acknowledgement}

This work was supported by the NUAA Fundamental Research Funds under Grant NZ2013201, the Fundamental Research Funds for the Central Universities NJ20130015 and National Nature Science Foundation of China under Grant No. 51175248/E050603. Professor Jun Wang of NUAA gives many helps for our work.

\section{References}

[1] Y.X. Chen, K. Zhang, Y.N. Hao and Y.C. Hu, Research status and application prospects of digital technology in orthopedics, Orthopedic Surgery 4 (2012), 131-138.

[2] B. Starly, Z. Fang, W. Sun and W. Regli, Three-dimensional reconstruction for medical-CAD modeling, Journal of Computer-Aided Design and Application 2 (2005), 431-438.

[3] Y. Jun and S. Park, Polygon-based 3D surgical planning system for hip operation, International Journal of Precision Engineering and Manufacturing 12 (2011), 157-160.

[4] M. Sonka, V. Hlavac and R. Boyle, Image Processing, Analysis, and Machine Vision, Academic Press, Boston, 2011.

[5] J. Lai and Q.J. Wei, Automatic lung fields segmentation in CT scans using morphological operation and anatomical information, Bio-Medical Materials and Engineering 24 (2014), 335-340.

[6] C.C. Lai and C.Y. Chang, A hierarchical evolutionary algorithm for automatic medical image segmentation, Expert Systems with Applications 36 (2009), 248-259.

[7] C.L. Alberola, M.F. Martuin and J.A, Ruiz, Comments on: A methodology for evaluation of boundary detection algorithms on medical images, IEEE Transactions on Medical Imaging 23 (2004), 658-660.

[8] Y. Cheng, S. Zhou a, Y. Wang et al., Automatic segmentation technique for acetabulum and femoral head in CT images, Pattern Recognition 46 (2013), 2969-2984.

[9] F. Shi, J. Yang and Y. M. Zhu, Automatic segmentation of bladder in CT images, Journal of Zhejiang University Science A 10 (2009), 239-246.

[10] K. Djemal, W. Puech, B. Rossetto et al., Automatic active contours propagation in a sequence of medical images, International Journal of Image and Graphics 6 (2006), 267-292.

[11] B. Veronica, V. C. Raquel and Y. S. Oscar, Automatic initialization for a snakes-based cardiac contour extraction, in: Proceeding of the $22^{\text {nd }}$ Annual EMBS International Conference, S. Demir, ed., IEEE Press, USA, 2000, pp. 1625-1628.

[12] Z.Wu, K.D. Paulsen and J.M. Sullivan, Adaptive model initialization and deformation for automatic segmentation of T1-weighted brain MRI data, IEEE Transactions on Biomedical 52 (2005), 1128-1130.

[13] A. Daoudi, S. Mahmoudi and M.A. Chikh, Automatic segmentation of the left atrium on CT images, in: $4^{\text {th }}$ International Workshop on Statistical Atlases and Computational Models of the Heart, O. Camara, ed., Springer-Verlag, Germany, 2013, pp. 14-23.

[14] S. Martijn, M. Henk and E.A. Hendriks, Automatic initialization algorithm for carotid artery segmentation in CTA images, in: Medical Image Computing and Computer Assisted Intervention, J. Duncanand and G. Gerig, eds., SpringerVerlag, Berlin, 2005, pp. 846-853. 
[15] L. He, Z. Peng, E. Bryan et al., A comparative study of deformable contour methods on medical image segmentation, Image and Vision Computing 26 (2008), 141-196.

[16] R. A.Zoroofi, Y. Sato and T. Sasama, Automated segmentation of acetabulum and femoral head from 3-D CT images, IEEE Transactions on Information Technology in Biomedicine 7 (2003), 329-341.

[17] R.A. Zoroofi, T. Nishii and Y. Sato, Segmentation of avascular necrosis of the femoral head using 3-D MR images, Computerized Medical Imaging and Graphics 25 (2001), 511-521.

[18] Y. Sato, K. Nakanishi, H. Tanaka et al., A fully automated method for segmentation and thickness determination of hip joint cartilage from 3D MR data, International Congress Series 1230 (2001), 352-358.

[19] J. Ehrhardt, H. Handels and T. Malina, Atlas-based segmentation of bone structures to support the virtual planning of hip operations, International Journal of Medical Informatics 64 (2001), 439-447.

[20] F. Matthias, E. Jan and H. Heinz, Automatic Atlas-based contour extraction of anatomical structure in medical images, in: Computer Assisted Radiology and Surgery Proceedings of the 19th International Congress and Exhibition, U.L. Heinz, I. Kiyonari and D. Kunio, eds., Elsevier, USA, 2005, pp. 272-277.

[21] F. Yokota, T. Okada and M. Takao, Automated segmentation of the femur and pelvis from 3D CT data of diseased hip using hierarchical statistical shape model of joint structure, In: the 14th International Conference on Medical Image Computing and Computer Assisted Intervention, G.Z.Yang, ed., Springer-Verlag, Berlin, 2009, pp. 811-818.

[22] M.G. Jinich, P. Meer and V. Medina, Robust Retrieval of 3D Structure from Image Stacks, Medical Image Analysis 3 (1999), 21-35.

[23] X. Hu, J. Xi and Y. Jin, Analysis and research of computer-aided model of hip joint based on reverse engineering, Chinese Journal of Mechanical Engineering 16 (2003), 309-312.

[24] F.L. Derek, Analytical Methods of Optimization, Dover Publications Inc., USA, 2006.

[25] T. Okada, K. Yokota, M. Hori and M. Nakamoto, Construction of hierarchical multi-organ statistical atlases and their application to multiorgan segmentation from CT images, in: the 13th International Conference on Medical Image Computing and Computer Assisted Intervention, D. Metaxas, ed., Springer-Verlag, Berlin, 2008, pp. 502-509.

[26] H. Liu, G.H. Zhu, J. Zhao et al., Recognition of occlusions in CT images using a curve-based parameterization method, International Journal of Image and Graphic 3 (2013), 186-212.

[27] S. Liu and W. Ma, Seed-growing segmentation of 3-D surfaces from CT-contour data, Computer Aided Design 31 (1999), 517-536. 